\title{
Proposed BISOL Facility - a Conceptual Design
}

\author{
Yanlin $\mathrm{Ye}^{1}$ (for BISOL Collaboration),* \\ ${ }^{1}$ State Key Laboratory of Nuclear Physics and Technology, School of Physics, Peking University
}

\begin{abstract}
In China, a new large-scale nuclear-science research facility, namely the "Beijing Isotope-Separation-On-Line neutron-rich beam facility (BISOL)", has been proposed and reviewed by the governmental committees. This facility aims at both basic science and application goals, and is based on a double-driver concept. On the basic science side, the radioactive ion beams produced from the ISOL device, driven by a research reactor or by an intense deuteron-beam accelerator, will be used to study the new physics and technologies at the limit of the nuclear stability in the medium mass region. On the other side regarding to the applications, the facility will be devoted to the material research associated with the nuclear energy system, by using typically the intense neutron beams produced from the deuteron-accelerator driver. The initial design will be outlined in this report.
\end{abstract}

\section{Brief history}

Around the year 2010, China Institute of Atomic Energy (CIAE) and Peking University (PKU) had separately proposed two ISOL-type facilities, namely "China Advanced Rare Ion beam Facility (CARIF)" and "Intense multi-beam multi-PUrpose Facility (ImPUF)", respectively. In 2011, CIAE and PKU signed an agreement to jointly promoting an ISOL-type RIB facility in Beijing area. As a result the previously proposed CARIF and ImPUF were merged into a combined project "Beijing Isotope-Separation-On-Line neutron-rich beam facility (BISOL)".

In October 2012 an international advisory committee (IAC), led by Professors Shoji Nagamiya and Jiaer Chen, was formed and a review meeting was held at PKU to evaluate the "Initial Conceptual Design of the BISOL". The committee gave a high appraisal to the proposal and "considers the research potential of the proposed facility in both, basic as well as applied and interdisciplinary research, as excellent and highly competitive on the world level. It promises a unique science reach in several respects, in particular with regard to the most neutron rich exotic nuclei and the study of the astrophysical r-process."

In 2013, the advanced ISOL-type facility was adopted in "the national mid- and longrange plan ( till 2030) of the major facilities for science and technology development".

In August 2014, a Xiang Shan Forum was successfully organized and a road map for major nuclear physics research facilities was established, including the BISOL as the nextgeneration major facility.

Then in May 2016, a domestic expert meeting was held at CIAE to evaluate the preparation works of the BISOL, aiming at a proposal to the 13th 5-year plan of the central government of China. Soon after, the project was included into a list of the large-scale-facility

\footnotetext{
*e-mail: yeyl@pku.edu.cn
} 


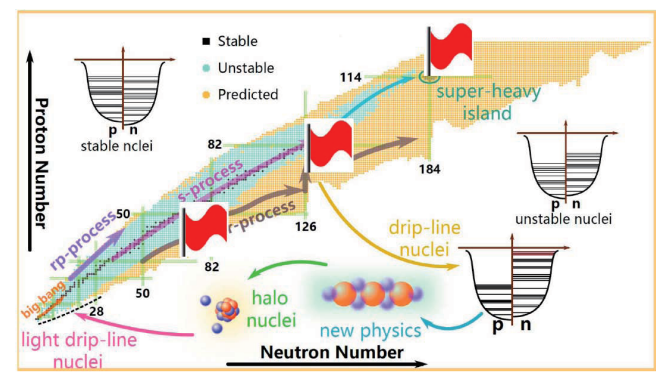

Figure 1. Great scientific questions at the expanded nuclear chart (see the text for details).

proposals and reviewed by the National Development and Reform Council in order to establish the priorities for the coming 5-year plan. As a result the BISOL proposal was successfully classed as one of the preparation facilities.

In January 2017, another topical IAC meeting was held at PKU-CIAE, which was dedicated to the accelerator based intense neutron source, in particular the high-power target systems. The first BISOL user meeting was also held at PKU in March with 150 participants who enjoyed active discussions. Then in June, the conceptual design of BISOL (BISOL-CD1) was finalized and evaluated by an domestic expert committee, and was ready for the next national review and the next IAC review.

\section{Background in basic and applied sciences}

The BISOL aims at both the basic nuclear science research as well as the nuclear technique applications. The basic scientific questions at the expanded nuclear chart, which will be addressed by BISOL, include: the new physics at the drip-lines; the nuclear-processes in creating heavy elements in the stars; the ways towards the stable island for super-heavyelements. Fig. 1 gives an illustration of these major scientific goals. At the application side, BISOL will be dedicated to meet the urgent needs for applying the intense neutron beams, such as studying the mechanism of the neutron irradiation damages of the materials, the material evaluations for the nuclear-energy systems; the nuclear reaction data with fast neutrons; and so on. These applications are extremely important considering the planned tremendous growth of the nuclear power in China in the coming 30 years or so.

The requirement of RIBs for studies of unstable nuclei has been outlined previously [1], based on which we have defined the primary parameters and the basic configuration of the BISOL, as illustrated in Fig. 2. The idea is to combine the reactor driver (RD) and the intense deuteron-beam driver (IDD) to produce the isotopes, to apply both the isotope separation on line (ISOL) and the projectile fragmentation (PF) techniques (the latter follows the former) in order to obtain the most neutron-rich RIBs in the medium mass region, and to target both the basic science problems as well as the key application problems. This interwinding concept is quite in line with the traditional Chinese Dao-philosophy which emphasizes the combination of various aspects into one unit, as symbolized by the "Taiji picture" in the inset of Fig. 2. The reason to have two drivers is basically the complementarity. The deuteron-beam driver (IDD) should produce fast neutrons with energies up to $20 \mathrm{MeV}$ while the reactor driver (RD) provides neutrons with much lower energies $(\sim 1 \mathrm{MeV})$. The higher-energy-neutron induced fission leads to more uniform isotope yields over the medium-mass region, whereas the low-energy neutron would induce fission-fragments focus on the two fission-fragment 


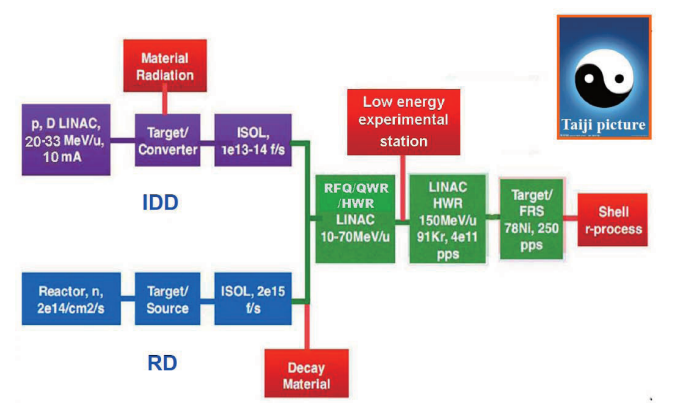

Figure 2. Primary parameters and the basic configuration of the BISOL. The inset at the upper-right corner shows a picture of the traditional Chinese Dao-philosophy, which symbolize the current design concept of combining various goals and technologies.

peaks. Therefore two drivers allow to produce high-intensity beams at different mass region. More importantly, these two drivers can be operated simultaneously to double the machine efficiency. For instance when the RD drives the ISOL target for the basic research purpose, the IDD may be used separately to irradiate materials for the application purpose. These dual-purposes are essential for the BISOL-design.

\section{Introduction to the conceptual design}

The overall design of the BISOL is displayed in Fig. 3. It is composed of the reactor driver (RD) using the existing China Advanced Research Reactor (CARR) and the intense deuteron linear accelerator driver (IDD, D-LINAC) together with the respective target systems, the low energy ISOL devices to separate the selected isotopes, the post-acceleration up to $150 \mathrm{MeV} / \mathrm{u}$, the projectile fragmentation followed by the online separation of the very neutron-rich nuclei via a high-power separator (FRS).

The experimentation is configured into three areas shown in Fig. 3: the Separation Energy Experiments with beams at 20keV/q (SEE); the Low Energy Experiments with beams at 20 $\mathrm{MeV} / \mathrm{u}$ (LEE); and the Intermediate Energy Experiments with beams at $150 \mathrm{MeV} / \mathrm{u}$ (IEE). Various instrumentations will be built in these areas.

Fig. 4 shows a more realistic placement of the facilities. Due to the limited land available to the facility, two levels (floors) arrangement is considered, with the high-intensity deuteron accelerator and the LE-ISOL together with the LEE placed in the underground floor, and the post-acceleration together with LEE and IEE placed at the ground floor.

The parameters for the isotope yields and the RIBs are specified in Figs 3 and 4. The estimated intensities for the typical ISOL-type beams are listed in Table 1. The combination of the applied technologies allows to produce the double-magic nucleus ${ }^{78} \mathrm{Ni}$ and the neutrondrip-line nucleus ${ }^{120} \mathrm{Sr}$ as high as 250 and $2 \times 10^{-4} \mathrm{pps}$, respectively, based on the simulation calculations. These reported numbers are obtained based on the reactor-driver (RD) configuration. The neutron flux is about $8 \times 10^{14} \mathrm{~cm}^{-2} \mathrm{~s}^{-1}$ and a $5 \mathrm{~g}{ }^{235} \mathrm{U}$-target is assumed, resulting in a fission rate of about $2 \times 10^{15} \mathrm{~s}^{-1}$. Similar fission-rate is also estimated by using the deuteron-beam driver (IDD).

On the application side, BISOL will be used to study material properties under strong neutron irradiation. The deuteron-LINAC can be operated independently to generate highflux neutrons. The liquid lithium will be used as target for neutron production. The target must sustain a beam power of $400 \mathrm{~kW}$. The estimated neutron irradiation capabilities depend 


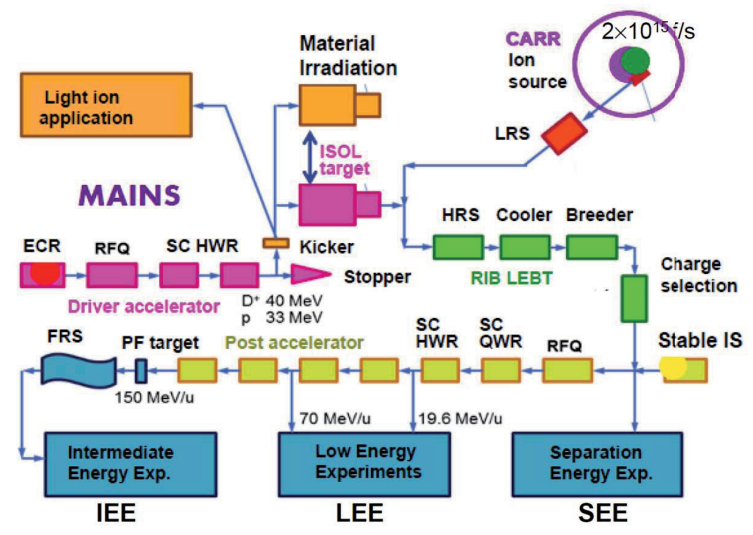

Figure 3. Specifications of various parts of the BISOL, together with the basic designed parameters.

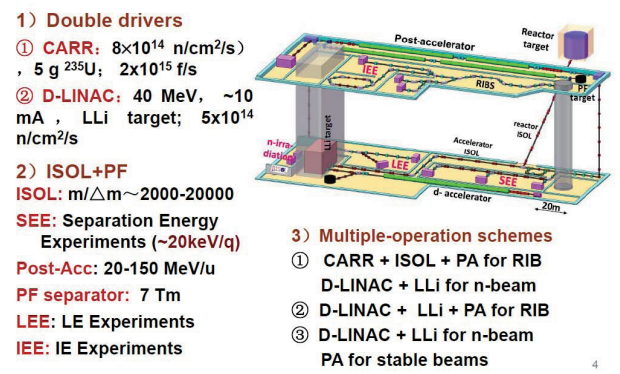

Figure 4. Schematic view of the arrangment of the BISOL facility. The basic parameters of the majors are listed.

Table 1. Estimated beam intensity

\begin{tabular}{|c|l|l|l|l|l|l|}
\hline nuclei & Fis. yield & rate & Target + isol eff. & CB eff. & Linac eff. & intensity \\
\hline${ }^{91} \mathrm{Kr}$ & $3.2 \times 10^{-2}$ & $6.4 \times 10^{13}$ & $13.0 \%$ & $10 \%$ & $50 \%$ & $4 \times 10^{11}$ \\
\hline${ }^{142} \mathrm{Xe}$ & $4.3 \times 10^{-3}$ & $8.8 \times 10^{12}$ & $2.0 \%$ & $10 \%$ & $50 \%$ & $9 \times 10^{9}$ \\
\hline${ }^{132} \mathrm{Sn}$ & $5.7 \times 10^{-3}$ & $1.2 \times 10^{13}$ & $8.0 \%$ & $10 \%$ & $80 \%$ & $7 \times 10^{10}$ \\
\hline${ }^{81} \mathrm{Ga}$ & $7.6 \times 10^{-5}$ & $2.0 \times 10^{11}$ & $8.0 \%$ & $10 \%$ & $95 \%$ & $1 \times 10^{9}$ \\
\hline
\end{tabular}

on the distance from the target and are illustrated in Fig.5. In the most favorable case (central region) 8-15 displacement per atom (dpa) can be achieved for one full power year (fpy) of irradiation, which is already the highest record at the near-future accelerator facilities and is very useful for material studies. This part of the facility is also called "the Multi-purposeAimed Intense Neutron Source" (MAINS in Fig. 3).

The detailed design has been performed for each part of the facility, including: (1) the reactor target; (2) the deuteron-LINAC; (3) the high power target and material irradiation station; (4) the low energy ISOL; (5) the separation energy experiments (SEE); (6) the postacceleration; (7) the low energy experiments (LEE); (8) the PF separator and the intermediate energy experiments (IEE). The infrastructure is also designed by the an expertized company. 


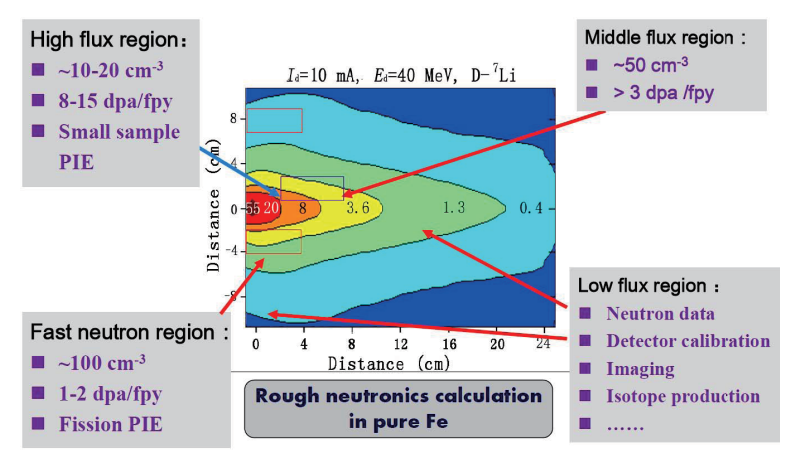

Figure 5. Irradiation performances at the target station down stream of the deuteron LINAC.

All details of the conceptual design version-1 are presented in an internal report BISOL-CD-1 (June 2017) [2].

The land for BISOL installation has already been allocated by CIAE, located at the central region in the CIAE campus near the CARR reactor. This is extremely important considering the safety and environment control for the strong-irradiation apparatus.

\section{Summary}

BISOL, in combination of the ISOL production through either the high-intensity deuteron beam driver or the high thermal neutron-flux from the reactor, together with re-acceleration of RIBs up to projectile-fragmentation energies (i.e. 150 AMeV), promises the most intense RIB capabilities in the medium mass region. The availability of accelerator-driven high-flux neutrons, produced with an intense beam of $40 \mathrm{MeV}$ deuteron, provides for a wide spectrum of applied research over a range of energies, in particular for unique material research relevant to the next generation fission and fusion reactors.

The facility cost is estimated to be about 3.5B RMB ( 500M USD) plus the infrastructure and manpower investment. The timetable is not clear yet but we expect a completion time in 2025-2030.

\section{ACKNOWLEDGEMENTS}

The author wish to thank all members of the BISOL collaboration who have contributed to this conceptual design. Particularly the original figures presented here were drawn by Dr. Jian GAO, Weiping LIU, Zhiyu GUO, Yang Liu, Biao YANG, Yugang WANG et al., and should be acknowledged. This work is partially supported by the 973 Program of China under Grant Nos. 2013CB834400 and 2014CB845500.

\section{References}

[1] Baoqun Cui, Yuan Gao, Yucheng Ge, Zhiyu Guo, Zhihong Li, Weiping Liu, Shixiang Peng, Zhaohua Peng, Zhi Wang, Sha Yan, Yanlin Ye, Sheng Zeng, Guohui Zhang, Feng Zhu, Nucl. Instr. Meth. in Phys. Res. B 317,257-262(2013).

[2] BISOL collaboration, Internal report BISOL-CD-1 (June 2017). 